\title{
ELEMENTOS CLÁSICOS EN LAS NOCHES DEL BUEN RETIRO DE PÍO BAROJA
}

\author{
Francisco Salas Salgado \\ Universidad de La laguna \\ frasalas@ull.es
}

TIBI, ISABELLA!, CATVLLO DVCE,

OPVSCVLVM NOVVM LEPIDVM (VT SPERO) DONO

\begin{abstract}
RESUMEN
La novela Las noches del Buen Retiro de Pío Baroja guarda una relación intrínseca con el tópico de la fugacidad de la vida. Pero además contiene toda una serie de elementos que tienen que ver con al mundo y la cultura clásica. En este trabajo intentamos destacarlos.

Palabras Clave: Tradición clásica, Generación del 98, Pío Baroja.
\end{abstract}

\section{ABSTRACT}

"Classical Elements in Las Noches del Buen Retiro by Pío Baroja». The novel Las noches del Buen Retiro written by Pío Baroja has an intrinsic relation with the topic of the fugacity of the life. But it also contains many parts that have to do with the world and the classical culture. In this paper we try to highlight them.

KEY wORDS: Classical Tradition, Generation of '98, Pío Baroja.

1. Determinadas obras que, por su título o por la época en que fueron escritas, parecen presagiar de antemano que nada tienen que ver con determinadas parcelas de estudio, luego suscitan sorpresas inesperadas. Ocurrió esto cuando revisaba algunos tomos de autores que fueron lectura de obligado cumplimiento en la época ya lejana de los estudios secundarios — posteriormente se convertirían algunos de ellos en dilectos-. Una de estas obras, releída casi de un tirón, fue Las noches del Buen Retiro de Pío Baroja, uno de los escritores, como es sabido, que se incluye en la denominada "Generación del 98».

Cierta formación (ya quizás deformación) profesional hizo que este nuevo acercamiento lo hiciera con un nuevo enfoque: lo que en épocas pasadas resultaba ser un hecho sin excesiva trascendencia o quizás resultara impertinente, ahora iba a centrar mi atención. Me refiero a ciertas partes de esta novela que tienen que ver con la pervivencia de la cultura clásica, esa especie de pilar (o lugar común) que de una forma u otra sustenta el armazón literario y cultural de países diversos y logra unificar las diferentes épocas. 
2. Puestos a indagar desde esta perspectiva me puse a la búsqueda de referencias que sirvieran para dar soporte material a este trabajo. Evidentemente sobre Baroja se ha escrito mucho. Basta ojear las diversas historias de la literatura española para disponer de un arsenal de investigaciones al respecto con referencias diversas. Para las pretensiones de este trabajo considero suficientes los datos que se refieren en algunas de esas historias, que mayormente se repiten en todas. Así hay mención a su nacimiento, ocurrido en San Sebastián el 28 de diciembre de 1872, a su familia, a una infancia no dichosa con el desarraigo que produce ir de un lado a otro; pero muy poco, más bien nada, se dice de sus primeros estudios y de sus estudios secundarios, y algo, y no mucho, de sus estudios universitarios, de una carrera de Medicina que casi no ejerció. Tampoco en relación con la producción literaria de Baroja existe uniformidad, solo en algunas de estas se presta una atención particular a sus obras, clasificándolas y atendiendo al contenido y significación de las mismas ${ }^{1}$, entre ellas la novela que aquí se va a tratar, aunque la información sobre la misma es escasa².

De igual forma son insuficientes las noticias que he podido reunir sobre la formación clásica de este autor, sobre el aprendizaje y la enseñanza de las lenguas clásicas, que sabemos seguía vigente ${ }^{3}$. La única mención (y tampoco es gran cosa) relacionada con su formación en estas materias la refiere Sebastián Juan Arbó (1963: 43) cuando nombra, junto al profesor de aritmética de Baroja en San Sebastián, Gregorio Pano, a un anónimo profesor de latín, menos dado a amonestar al joven Pío, como hacía el profesor de aritmética, quien por otro lado no tenía muy buena consideración de su alumno:

Al lado de don Gregorio estaba el profesor de latín. Éste, según él, no le riño nunca, pero le envió dos veces a la corrección. La corrección era - dice- un cuartucho con rejas a manera de calabozo, en donde en invierno se tiritaba de frío. Éste no era aficionado a anunciarle glorias futuras. Era peor: el que no salía por un lado, salía por el otro.

\footnotetext{
${ }^{1}$ Así Pedraza-Rodríguez (1987). He manejado, además, Domingo (1982), Shaw (1992) y Mainer (1980, 2012).

${ }^{2}$ Tengo que referirme de nuevo a Pedraza-Rodríguez (1987: 473), quien aporta, aunque solo sea en media página, datos del argumento de las Noches del Buen Retiro, precisando que el título de esta obra obedece a que en ella se describe el ambiente del Madrid de principios de siglo que conoció Baroja en sus años juveniles. Quizás esto se deba a la mayor fama que obtuvieron algunas de las obras de Baroja en detrimento de otras. En esto insiste Domingo (1982: 83), para quien la trilogía en la que está inscrita esta novela (La juventud perdida) junto con Agonías de nuestro tiempo, La selva oscura y Los visionarios, poco tiene que "pueda parangonarse con los grandes aciertos del novelista». Sin embargo Mainer (1980: 337) apunta que con Las noches del Buen Retiro Baroja superó la crisis creadora de La selva oscura, volviendo a la agridulce bohemia finisecular.

${ }^{3}$ Cf. García Jurado (2015).
} 
Sin embargo, parece, como señala J. A. López Férez (1998: 442, n. 19), que Baroja fue buen lector de clásicos grecolatinos ${ }^{4}$. Quizás esto se debió a la formación que recibiera no solo en su lugar natal sino especialmente en Madrid, donde fue alumno del Instituto de San Isidro, centro en el que terminó el Bachillerato5.

Asimismo, por lo que he podido averiguar, son escasos los trabajos que atienden a la pervivencia clásica que existe en su obra. Solo he encontrado referencia a la mitología en el trabajo titulado «El laberinto de la sirenas», que se debe a M.a Ángeles Durán (2010).

3. Así las cosas, conviene ya entrar ya en materia. Las noches del Buen Retiro fue una novela escrita en 1934. Pertenece a una de las nueve trilogías en que Baroja dividió su obra, la que intituló, "La juventud perdida», en la cual también se encuentran El cura de Monleón (1936) y Locuras de carnaval (1937).

Las noches tienen un personaje central, Jaime Thierry, que aparece en el capítulo XI, escritor formado en Francia y en Estados Unidos, que fue director de uno de los periódicos más críticos que se nombra en el relato, denominado «El Bufón». Thierry empieza causando sensación y no pocas antipatías en quienes veían en él una especie de lord Byron, un bohemio no tradicional sino revolucionario, dotado de un individualismo radical. Esta actitud inicial, arrogante, se difumina a lo largo de las páginas cuando el personaje poco a poco va cayendo en la miseria, enlazando una desgracia tras otra, hasta enfermar y morir a causa de la tuberculosis (detrás de todo esto se esconde la pesadumbre de no verse querido por la mujer que amaba).

Jaime Thierry tenía una cultura elevada para la época, pero era de carácter difícil, costumbres obsesivas y neuróticas. Estaba imbuido en las teorías de Nietsche y destacaba por ser una persona hipercrítica (utilizaría el periódico para realizar una censura del ambiente en el que se mueve). En la novela mantiene varias relaciones sentimentales, sobre todo una turbulenta - la más comentada y la que le va a destrozar a la postre- con la condesa de Villacarrillo, cuya actitud más equilibrada y tranquila sirve de contrapunto al temperamento de Thierry.

${ }^{4}$ Vuelve a insistir López Férez (2008: 183) sobre esto en otro trabajo, destacando la presencia de Aristófanes en algunas secuencias de las obras de Pío Baroja. Aquí también aparecen otros autores y obras griegos leídos por Baroja, entre ellos la Odisea de Homero y Eurípides.

${ }^{5}$ Fue conocido como Colegio Imperial de Madrid o de los Reales Estudios de San Isidro. Fundado en 1566 por los jesuitas, se intentó convertirlo en Universidad en el siglo XVII aunque no obtuvo el fruto esperado por la enérgica oposición de las Universidades de Alcalá y Salamanca. En 1725 Felipe $\mathrm{V}$ funda dentro del Colegio un Real Seminario de Nobles. Datos generales sobre el mismo pueden verse en Simón Díaz (1992). Cf. sobre la enseñanza de las lenguas clásicas, aunque referido al siglo XVIII, Bartolomé Martínez (1995). 
Sin embargo, esta novela va mucho más allá y se convierte en un retrato personal de la sociedad del momento, sociedad caracterizada por la bohemia y la apariencia, por personajes atormentados, contrapuestos y extravagantes, donde la diferencia entre ricos y pobres estaba muy marcada, y donde el nivel cultural distaba mucho de ser relevante. Un lugar destacado en la novela lo ocupan las tertulias, que se dedicaban sobre todo a favorecer la murmuración y la insidia. Y en medio de todo esto, al hilo del relato, aparecen referencias, no muchas, a la cultura del momento y en particular al mundo clásico.

4. Rasgo destacado de esta novela es que usa uno de los mecanismos recurrentes en la ficción narrativa, «el del manuscrito encontrado o dado al autor», quien desde ese momento deja de ser autor y adopta el papel de editor. Esto se refiere en el «Capítulo I». Esta novela le había sido entregada a él, que había sido «un poco médico, un poco industrial, un poco negociante y un poco periodista" $\mathrm{y}$ director literario de una casa editorial (Baroja, 1982: 5), por una señora que vestía de luto, y que le pidió que considerara la publicación de un relato de un amigo suyo y la realización de un prólogo, pues el verdadero autor (a quien sus amigos conocían por el nombre de Fantasio) no quería que apareciera su nombre.

A los pocos días vino a su despacho un hombre pálido, también de luto —el editor pensó que sería el tal Fantasio_- quien le traía dicho manuscrito. Tras su lectura, consideró interesante publicar esa novela, aunque vacilara al principio, insistiendo de nuevo en que «el autor de la obra no soy yo, sino el señor misterioso, llamado por amigos Fantasio, y que usaba melena y chalina flotante y azul» (Baroja, 1982: 8), diferentes ambos en sus ideas y aficiones.

Esta suerte de preámbulo da paso al relato, supuestamente de Fantasio (capítulos II-LXXV), en el que, como se dijo, aparecen retratados muchos personajes de la época, sus actitudes y una sociedad clasista y aparente.

$\mathrm{Al}$ frente del libro este «caballero romántico de la barba y de la melena, antiguo joven tenebroso", puso una dedicatoria (la titula "A una señora amiga») que ocupa el capítulo II, donde concreta el motivo principal que rige esta novela y nos hace percibir claramente la causa de su inclusión en la mencionada trilogía.

En efecto, se habla aquí de la nostalgia del tiempo que ha pasado, de la época de la juventud, alegre, festiva y activa:

El pasado no es mejor que el presente... pero se alumbra con una media luz crepuscular sugestiva, poética, distinta a la claridad cruda y agria del momento (Baroja, 1982: 9).

Ese pasado, donde ambos eran jóvenes, tenía un escenario que los unía: los Jardines del Buen Retiro. Y reitera:

Y ahora los dos somos viejos. ¡Qué pena! Yo, pase; ¡pero usted! El escenario y los actores desaparecieron. El jardín ya no existe. La mayoría de nuestros amigos 
murieron. El tiempo ha volado como vuelan todos los tiempos. Hora fugax. Fugit irreparabile tempus (Baroja, 1982: 9) ${ }^{6}$.

Esta idea que guía la novela de principio a fin se ve acompañada de otras alusiones al mundo clásico, aunque habría que volver a insistir que no aparecen de forma continua. Veámoslas.

4.1. La enseñanza de las lenguas clásicas seguía su curso mal que bien. En la novela aparece algunos personajes que viven del latín (o mejor, malviven) pues aprovecharían sus conocimientos en la lengua del Lacio para ello. Era el caso de Emilio Aguilera, asiduo participante de una de las tertulias más nombradas entre la sociedad madrileña de entonces, la de Paco Lecea, quien simultaneaba su oficio de periodista con su empleo de profesor de latín y literatura en un colegio (Baroja, 1982: 22).

Parece que saber latín daba cierto empaque en esos momentos, ante determinadas concurrencias, aunque también no dejaba de tener cierta afectación. En el capítulo III en la descripción de ciertas damas que acudían a Los Jardines del Buen Retiro, todas sin maquillar, aparece la oportuna cita usada casi como elemento diferenciador de clases:

También entre las mujeres existía mayor variedad; casi ninguna se pintaba, o si lo hacía no era de una manera tan exagerada como en esta época. El maquillaje se consideraba sólo para las hetairas, para las horizontales, era la palabra del tiempo, y se miraba como algo chocante y de poca distinción.

La pintura desvergonzada y con mucha pasta de color sobre la piel de la cara y la depilación de las cejas ha dado a las mujeres de hoy un aire inexpresivo de muñecas y una falta absoluta de carácter. Princesas, manicuras, tanguistas y cocineras, todas parecen actualmente lo mismo, de la misma harina, ejusdem farinae, que decimos los latinistas (Baroja, 1982: 12).

La relevancia que daba soltar el latinajo de turno aparece también en otra parte de la novela, esta vez en boca de uno de los personajes que frecuentaban esas tertulias. El personaje en cuestión es Juan de Guevara, médico solterón, culto y de buena posición, quien en una de sus frecuentes pláticas describía tres clases de hombres de la siguiente manera: «el Homo Sapiens, raro; el Homo Demens, corriente, y el Homo Domesticus o Vulgaris, frecuentísimo» (Baroja, 1982: 22). Debía tener fama este Guevara de pasarlo todo al latín, como se puede comprobar cuando relata Baroja

${ }^{6}$ Cf. Verg. Georg. 3, 284. La pérdida de la juventud tiene mucho que ver con lo que comenta luego sobre la fuente de Juvencio (Fons Iuuentutis) «que el marino español Ponce de León creyó encontrar en La Florida» (Baroja, 1982: 10). 
las pretensiones a que aspiraba Fernanda Arias Mejía, una acomodada damisela que frecuentaba los Jardines, en sus relaciones de pareja (en este caso referidas a Carlos Hermida ${ }^{7}$, quien en busca de una sólida posición, la pretendía):

No aspiraba a un marido brillante; le veía a Carlos como a un joven de quien se puede sacar partido. Era para ella el tipo medio del hombre, del cual una mujer puede esperar una vida tranquila y relativamente feliz, el homo domesticus, como hubiera dicho el doctor Guevara (Baroja, 1982: 120).

El propio Jaime Thierry también emplea en una ocasión alguna expresión latina. Ocurre cuando conoce a Jacinto Palacio del Campos, asturiano que había estado en Cuba, en La Habana, y fue acusado de malversador. Su intención era crear un periódico (que se llamaría el «El Bufón») y propone a Thierry que lo dirija:

Bien —dijo don Jacinto—; pero, aunque sea así, haremos el periódico y usted lo dirigirá.

-Bueno, bueno. Estoy conforme.

- Además, amigo Thierry, le pagaré lo que usted me diga.

- No me opongo; ante estos argumentos ad hominem hay que ceder (Baroja, 1982: 168).

Pero no solo los que habían podido disfrutar de estudios superiores usaban el latín. Jaime Thierry estimaba mucho la memoria de su madre, supo que vivía en Madrid la hija de un capataz que había trabajado para la familia de su madre en

${ }^{7}$ El ambiente cultural del momento ofrece personajes como este. Carlos Hermida, también periodista, era hijo de un empleado de la Sección de Fomento ya muerto, cuya madre doña Antonia se preocupó mucho para lograr el ascenso social de su hijo. Hermida había logrado terminar el bachiller, pero por falta de medios no había podido hace una carrera. Mal estudiante en Bachillerato, no manifestaba afición a ciencia o arte, ni siquiera a hacer versos, solo le interesaba la política y tener cierta posición social. Su ascenso en el mundillo periodístico se lo debió a su madre y a su novia Matilde Leven, aficionada a la literatura y buena escritora de versos y cuentos, de cuyo ingenio y creatividad se benefició Carlos (resulta llamativo que ella no quería publicar nada con su nombre pues «de una mujer sabia y literata se ríe en España todo el mundo" [Baroja, 1982: 32]). Carlos Hermida solía frecuentar la tertulia de un café del comienzo de la calle de Alcalá, próximo a la Puerta del Sol, donde se reunían literatos, poetas, dramaturgos y dos o tres aprendices de novelistas. El conocimiento de estos de la literatura, de lo que hablaban siempre, se circunscribía a los autores de su época, sobre todo extranjeros, los autores de principios de siglo, pero los del XVI y XVII les eran desconocidos y «muy pocos sabían algo de literatura antigua» (Baroja, 1982: 45), si bien se debe referir a los clásicos castellanos, aunque tampoco habría que obviar a los grecolatinos. En contrapartida Jaime Thierry, un extranjero venido a España, sintió desde temprano, a pesar de sus ideas filosóficas, pasión por la literatura clásica española. Cuando se fue a vivir a las afueras, al hotelito que le habían encontrado, «se dedicaba por entonces a los autores castellanos clásicos y modernos y a tomar sus notas. Gonzalo de Berceo y el arcipreste de Hita eran sus escritores favoritos. Era también entusiasta de fray Luis de León, de San Juan de la Cruz, de la novela picaresca y de los dramas de Calderón» (Baroja, 1982: 58). 
Burgos. Esto le llevó a conocer a la Silvestra y a su marido Beltrán. Beltrán era hijo del sacristán del pueblo y había comenzado a estudiar para cura, pero la falta de vocación se lo impidió. Sin embargo «todavía recordaba algunos latines, sobre todo macarrónicos y de aire pedantesco, pero más que los latines le gustaba el argot popular» (Baroja, 1982: 53).

4.2. Otras muestras de pervivencia de la cultura grecolatina existen en esta obra, que aparecen de forma esporádica en menciones puntuales. Puestos a hacer una clasificación de estas habría que referirse en un primer momento a referencias mitológicas e históricas, de figuras y personajes, que la tradición ha tipificado como símbolos de determinadas virtudes y vicios o de determinadas situaciones, y luego las realizadas sobre escritores de la Antigüedad.

4.2.1. Un primer ejemplo dentro del primer grupo lo tenemos en la expresión «espada de Damocles» usada por Baroja para referirse al hecho de que la gente pobre y sin pretensiones en los meses de verano no podía desplazarse a la costa cantábrica, lo que hacía que fueran mal mirados, «a la cual hoy parece írsele quitando la punta y filo" (Baroja, 1982: 11).

Curiosa es también la referencia a la diosa de la belleza que aparece en el capítulo IV. La diversidad de personajes de diferentes clases sociales que frecuentaban los Jardines del Buen Retiro le lleva a hablar de las cortesanas, que también habían, de «la Blanca, la Puri, la Tropical, la Nadadora, Lola la Valkiria», y la que llamaban «la Venus de la Necrópolis, sea porque tuviera citas en los cementerios o porque se la considerase a ella como sepulcral» (Baroja, 1982: 18).

Una figura mitológica es la aparece en los motes que la gente mundana y de perversa intención había dado a tres señoritas de clase acomodada que acompañaban a Fernanda Arias Mejía. Estos nombres por otro lado los tenían tres torpederos de la marina de guerra española, a saber Terror, Furor y Proserpina (los que andaban tras ellas para cazar su dote se les apodaba con el nombre de tres cañoneros o barcos de guerra: el Audaz, el Osado y el Temerario) (Baroja, 1982: 119). Otra referencia a la mitología se encuentra también en «dos estatuas de piedra, una de Flora y otra de Pomona» (Baroja, 1982: 55) que adornan la puerta del mal conservado hotel con un jardín cercado con sus tapias, adonde había ido a vivir Thierry.

Este uso de figuras mitológicas con una función referencial o sarcástica continúa en el relato de Baroja esta vez en la figura del Barquero de la Estigia, que aparece en uno de los diálogos que Jaime Thierry realiza en el primer número del periódico «El Bufón» del que era su director. Caronte conversa allí con un vendedor de petróleo, y se quejaba de la carestía del preciado líquido para el motor de su barca «y de que muchas almas de los muertos daban moneda falsa o no pagaban los billetes del trayecto por la laguna Estigia porque no tenían un cuarto» (Baroja, 1982: 176).

4.2.2. Por el contrario, los personajes históricos (o que tienen alguna relación con la historia) se usan en una función paradigmática. Lo encontramos por ejemplo en la mención a Lucrecia o Cornelia, que han pasado a la posteridad como modelo de 
virtud $^{8}$. Ciertamente, la desazón que empezó a tener Jaime Thierry tras romper con Josefina Cuéllar e iniciar la relación con Concha Villacarrillo, originada por los celos que aquella empezó a sentir ante los comentarios que se hacían de esta, debido al carácter alegre y dispuesto de la condesa, le lleva a reflexionar que hubiera preferido «que la considerasen como una mujer virtuosa, como una Lucrecia o la madre de los Gracos. Luego, ya comprendía la estupidez de esta pretensión» (Baroja, 1982: 129-30).

De otra parte está la figura de Creso que aparece en medio de una conversación donde se discute sobre la posibilidad o la necesidad de gastar la riqueza personal' . La discusión se establece en el entreacto de una de las funciones que ofrece el Teatro Real entre Alejandro Dobón y Eduardo Larraga, redactores del periódico «El Mundo»:

No todo el mundo puede gastar en duros, aunque los tenga —aseguró Dobón. —Todo eso no son más que ilusiones — replicó Larraga—: el poder gastar no depende de la mentalidad, sino del dinero.

-De las dos cosas. Hay personas que aunque fueran Cresos por su fortuna vivirían mezquinamente (Baroja, 1982: 146).

4.2.3. Los escritores clásicos también encuentran su acomodo en el relato barojiano. Aunque la mención de algunos de ellos debe ser tomada con cautela, porque no indica que se haya leído a los mismos, en Baroja parece que lo contrario.

Uno de los personajes que se quedaba en Madrid en la época de verano era el marqués de Quiñones, quien solía dejar a su familia en alguna playa del Norte para "irse de rositas" por la capital, frecuentando así los saloncillos de los teatros y los camerinos de las artistas. Al parecer el marqués tenía «un sosias», un cómico amigo suyo que lo imitaba en la manera de hablar y de vestir, y para quien el aristócrata eran un modelo a seguir. Y aquí surge la comparación. Dice Baroja:

Este cómico representaba los papeles de aristócrata en las comedias, sin duda por su parecido con el marqués, a quien se le consideraba un Petronio, un arbiter elegantiarum (Baroja, 1982: 80) ${ }^{10}$.

La crítica despiadada que se hacen entre sí estos personajes barojianos, incluso perteneciendo a la misma clase y grupo social, se refleja en muchos lugares. Jaime Thierry había sido invitado por un tal Alfredísimo (así es como se le denomina en la novela por su atildamiento y exquisitez, aunque se llamaba Alfredo de Mendoza,

${ }^{8}$ Recordemos los retratos que nos ofrecen Livio, 1, 57-59 y Plutarco, TG, 1, 3-6, respectivamente.

${ }^{9}$ Remito al relato de Heródoto, I, 29, y Plutarco, Sol. 27, 1-9; 28, 1-6.

${ }^{10}$ Esta expresión se encuentra en Tácito (ann. 16, 18): De C. Petronio pauca supra repetenda sunt. [...] Dein reuolutus ad uitia seu uitiorum imitatione inter paucos familiarium Neroni adsumptus est, elegantiae arbiter, dum nibil amoenum et molle adfluentia putat, nisi quod ei Petronius adprobanisset. 
era asiduo de tertulias y muy solicitado por las damas) a una cena que resultó realmente penosa. Las críticas, por ello, empezaron a surgir y el americano aprovecha para ensañarse con el carácter superfluo el español de entonces, sobre todo el de los débiles aristócratas, que prefiere gastar su dinero en convidar a tales individuos y no dispondría ni la cuarta parte de este «en convidar a Platón en compañía de Galileo y de Pasteur» (Baroja, 1982: 155) ${ }^{11}$, con lo que queda patente la crítica a la desidia cultural de entonces.

En el primer número de «El Bufón» Thierry empezó una sección que tituló «Diálogos de los muertos del siglo XIX», firmando con el seudónimo de Menipo (quizás por vinculación con el cínico Menipo de Gádara debido al carácter crítico y satírico que caracterizaría al periódico). Uno estos diálogos fue el de las cortesanas «a imitación de Luciano» (Baroja, 1982: 176). También aparecieron dos artículos muy celebrados de autor desconocido, «uno titulado “¡Viva todo el mundo!", y el otro "La apokolokyntose", imitado de Séneca, en donde un político convertido en calabaza vomitaba una cosa negra y fétida, que era su alma» (Baroja, 1982: 178).

Relacionado también con este periódico se encuentra una nueva mención de otro autor clásico. Los redactores del «El Bufón» acostumbraban terminar sus jornadas en una cervecería llamada «La Brasileña» (o en otros lugares y tugurios), donde simpatizaron con algunas de las camareras del local, chicas más o menos descarriadas, sacadas algunas de los burdeles. En una de esas ocasiones, una de ellas, la Magnolia, junto a un personaje al que decían Traganiños, invitó a Thierry y a otro redactor, Federico Golfín, a cenar en el baile del circo de Colón, quienes acudieron acompañados de dos de esas camareras. En ese lugar, además, «se habían dado cita todos los homosexuales de la corte». Después de bailar se fueron al ambigú, donde Magnolia les esperaba con cuatro pseudomujeres. Allí también estaba un ilustre articulista. La conversación que surge entre ellos, cuando quería la Magnolia presentarlos, y la alusión a un escritor griego paradigma de la homosexualidad es como sigue:

-Estos son los que hacen «El Sapo».

—Nosotros hacemos «El Sapo» y vosotras la carrera — contestó Golfín con ironía-. ¿A qué no sabéis vosotros en que os parecéis a Socrates?

La Magnolia sí lo sabía. Se sentaron todos y pidieron la cena. La presidencia de la Patro y de la Antonia molestaba a los amigos de la Magnolia. Se dijeron una porción de brutalidades y de frases de doble sentido. Golfín estuvo cruel. Por muy cínicos que fueron, las palabras de Golfín tenían que molestarles.

${ }^{11}$ El escritor clásico como referencia necesaria para afianzar un aserto aparece también en el "Capítulo XXXIX», donde se narra la fiesta a la que fue invitado Thierry por un grupo de aristócratas en una finca de Torrelodones. La fiesta se daba en honor a la hija de un marqués casada con un príncipe polaco. Thierry causó viva impresión entre los asistentes, especialmente al marqués, padre de la princesa, quien dijo de Thierry una frase lapidaria: «-El señor Thierry es un hombre correcto. Para él esto debía valer tanto como para Homero el llamar a uno de sus héroes el irreprochable» (Baroja, 1982: 152). 
Al terminar la cena, éste gastó una broma a aquellos socráticos, a aquellos mariposos, como les llamaba él, broma que no les hizo ninguna gracia (Baroja, 1982: 184-185).

4.3. Por último es latente, o quizás deliberado, la presencia de determinados tópicos heredados de la Antigüedad que cierran esta novela. Parece en este sentido obedecer esta obra de Baroja a la estructura de la "Ringkomposition", pues era el tópico de la brevedad de la vida la que abría la misma y otros dos tópicos se asoman en los momentos finales, que son los más trágicos.

El enamoramiento de Thierry hacia la condesa y el desprecio de esta que observa en su joven amante un tono de locura motivado por los celos, por sus quejas y demandas, hace que Thierry se despreocupe de su salud física y enferme de tuberculosis. Gracias a Beltrán recibe las atenciones de un doctor joven, Montoya, quien frecuenta luego la casa del americano. Son momentos de reflexión donde en las situaciones más inesperadas aparecen estos tópicos.

Una repentina recuperación de Thierry le vuelve un tanto más cercano a las personas que desde el comienzo ha tenido al lado, las que conviven en su casa. Establece así cierto vínculo con Beltrán, a quien ayuda en las labores de carpintería. Beltrán le empieza a dar lecciones de guitarra. En una de sus conversaciones parecer estar presente el tema que se transmite en el verso virgiliano labor omnia vincit (georg. 1, 145):

— Lo que me ha perdido es el dinero — decía Thierry—. Si hubiese tenido que trabajar para vivir, habría vivido mejor.

Creo que el trabajo es lo único decente de la vida. Lo demás no vale nada (Baroja, 1982: 215-216).

También en medio de la enfermedad, el deseo irrefrenable de ver a su amada, le hace ir al pueblo donde Concha pasa sus días con su marido y sus hijas. La imagen de familia feliz que se le presenta y la charla con el marido de Villacarrillo le llevan a aceptar que su relación había acabado. Llegó a su casa medio moribundo, pilló una bronquitis, lo cual empeoraba su salud. Le visitaron sus amigos, iba incluso el administrador de Villacarrillo para lo que se ofreciera. Una fase transitoria febril le llevaba a delirar en muchas ocasiones. Una mañana en que estaba más despejado de la fiebre habló con Montoya sobre su muerte. La imagen de la fama, asociada al escritor, de la que tanto han hablado los clásicos (recordemos solo Hor. carm. 3, 30, 1-9) ocupa parte de la conversación que tiene con el médico aunque Thierry ya sabía que su fin estaba cerca:

Una mañana, que estaba más despejado de fiebre que de ordinario, tuvo una explicación con Montoya sobre su muerte.

— ¿No le preocupa a usted, como escritor, la posteridad? —le preguntó el médico. —iLa posteridad! ¿Qué me importa la posteridad? Eso no es nada.

- Pero, hombre, antes no pensaba usted así.

- Ha vivido un lo más intensamente posible. Se acabó la salud, se acabó el dinero, se fue la mujer. No vale la pena de vivir. Ha venido el punto final. Está bien (Baroja, 1982: 244). 
5. Una vez más queda demostrada la presencia de elementos culturales grecolatinos en cualquier momento de la tradición literaria. Baroja no escapa a este supuesto, aunque la importancia de los mismos queda en un segundo plano ante la fuerza de un relato crudo y realista como el que nos ofrece esta novela. Evidentemente nos encontramos con elementos aislados que delatan la preparación clásica de su autor, y que muchas veces se introducen para dar empaque culto o como mecanismo diferenciador de clases.

Sería pretencioso concluir que Baroja asienta esta novela sobre los pilares de sendos tópicos literarios heredados de la Antigüedad que aparecen en la parte inicial y final de la misma, sin embargo no deja de sorprender que cierto eco de aquellos acompaña a esa profusión de personajes y a la disparidad de situaciones que se describen.

\section{REFERENCIAS BIBLIOGRÁFICAS}

Baroja, Pío (1982): Las noches del Buen Retiro, Editorial Orbis, Barcelona.

BARTOlOMÉ MARTíneZ, B. (1995): «Educación y humanidades clásicas en el Colegio Imperial de Madrid durante el siglo XVII", Bulletin hispanique 97, 1:. 109-155.

Domingo, J. (1982): “La prosa narrativa hasta 1936”, en J. M. a DíEZ Borque (coord.), Historia de la literatura española. Tomo IV, El siglo XX, Taurus, Madrid, 2a reimpr., pp. 71-121.

DURÁN LÓPEZ, M. DE LOS Á. (2010): «El mito clásico en La aldea perdida de A. Palacio Valdés y en El laberinto de las sirenas de Pío Baroja, en J. A. López Férez (ed.), Mitos clásicos en la literatura española e hispanoamericana del siglo XX, vol. 1, Ediciones Clásica, Madrid, pp. 57-66.

GARCíA JURADO, F. (2015): «Reinventar la antigüedad: los estudios clásicos a partir del siglo XVIII», Revista de Occidente 410-411: 113-132.

LÓPEZ FÉreZ, J. A. (1998): «Estudio sobre la influencia de la comedia griega en la literatura española», en J. A. LÓPEZ FÉrEZ (ed.), La comedia griega y su influencia en la literatura española, Ediciones Clásicas, Madrid, pp. 387-457.

López Férez, J. A. (2008): «Aristófanes, Lisistrata», en P. Hualde Pascual - M. Sanz Morales (eds.), La literatura griega y su tradición, Akal, Madrid, pp. 145-184.

MAINeR, J. C. (1980): «Modernismo y 98», en F. Rico (ed.), Historia y crítica de la Literatura Española, Crítica, Barcelona, pp. 332-338.

Mainer, J. C. (2012): Pio Baroja, Taurus-Fundación Juan March, Madrid.

Pedraza Jiménez, F. B. - M. Rodríguez CáCeres (1987): Manual de literatura española. IX. Generación de fin de siglo: Prosistas, Cénlit Ediciones, Navarra, pp. 393-510.

SHAW, D. L. (1992): Historia de la literatura española. El siglo XIX, Ariel, Barcelona, 10a ed., pp. 255-263.

Simón DíAZ, J. (1992): Historia del Colegio Imperial de Madrid: (del Estudio de la Villa al Instituto de San Isidro, años 1346-1955), Madrid, Instituto de Estudios Madrileños. 
